\title{
Malignant Pulmonary Vein Neoplasm
}

National Cancer Institute

\section{Source}

National Cancer Institute. Malignant Pulmonary Vein Neoplasm. NCI Thesaurus. Code C5383.

A malignant neoplasm involving the pulmonary vein. 